\title{
MolAR: Bringing Chemical Structures to Life with Augmented Reality and Machine Learning
}

\author{
Sukolsak Sakshuwong, ${ }^{1}$ Hayley Weir,, ${ }^{2,3}$ Umberto Raucci,, ${ }^{2,3, \#}$ and Todd J. Martínez, ${ }^{2,3, *}$ \\ 'Department of Management Science and Engineering, Stanford University, Stanford, USA. \\ ${ }^{2}$ Stanford PULSE Institute, SLAC National Accelerator Laboratory, Menlo Park, USA. \\ ${ }^{3}$ Department of Chemistry, Stanford University, Stanford, USA. \\ "Present address: Italian Institute of Technology Genova GE, Italy.
}

\begin{abstract}
Visualizing three-dimensional molecular structures is crucial to understanding and predicting their chemical behavior. Existing visualization software, however, can be cumbersome to use, and, for many, hand-drawn skeletal structures remain the preferred method of chemical communication. Although convenient, the static, two-dimensional nature of these drawings can be misleading in conveying the molecule's 3D structure, not to mention that dynamic movement is completely disregarded. Here, we combine machine learning and augmented reality (AR) to develop MolAR, an immersive mobile application for visualizing molecules in real-world scenes. The application uses deep learning to recognize hand-drawn hydrocarbons structures which it converts into interactive 3D molecules in AR. Users can also "hunt" for chemicals in food and drink to uncover molecules in their real-life environment. A variety of interesting molecules are pre-loaded into the application, and users can visualize molecules on PubChem by providing their name or SMILES string and proteins in the Protein Data Bank by providing their PDB ID. MolAR was designed to be used in both research and education settings, providing an almost barrierless platform to visualize and interact with 3D molecular structures in a uniquely immersive way.
\end{abstract}




\section{Introduction}

In 1953 James Watson and Francis Crick proposed the double-helix model as the threedimensional structure of DNA. Computational modeling software was not available at that time; instead, they assembled a structure using a set of cardboard cutouts, each representing the different chemical components (the four bases and other nucleotide subunits). The pair "shifted molecules around on their desktops, as though putting together a puzzle."1-2 Although quite rudimentary, this visualization tool allowed them to observe how the complementary base pairs fit together, linked by a hydrogen bond, to form the structure of a double helix. The discovery marked a milestone in the scientific history, pushing forward our understanding of the genome.

This example showcases the importance of visualizing the 3D spatial arrangement of molecules in understanding their chemical behavior. Since Watson and Crick's discovery, 3D visualization tools have made giant strides, driven by constant technological advances. Soon, chemistry modeling kits were widespread in schools and labs; this allowed chemists to build molecules using spheres and sticks, see the bond angles and molecular shape, and feel which bonds can bend or twist. $^{3}$ Nevertheless, building a molecule from scratch during a short chemistry lecture can be quite impractical and time-consuming, while building a biomolecule requires specialist engineering skills. The emergence of graphical modeling software provided the next leap in molecule visualization, allowing chemists to not only image, but also interact with the 3D arrangement of atoms within molecules. ${ }^{4}$ Visualization of dynamic trajectories offered a further advance still, letting chemists watch molecules evolve in time, which is essential in understanding reaction mechanisms. Molecular visualization is just one example of how scientific progress has greatly benefited from the technological revolution, and as new technology continues to be developed, scientists will continue to adapt their workflows to the new available platforms (Figure 1).

Perhaps the most exciting visualization technology that has emerged in recent years is augmented reality (AR), which superimposes computer-generated content onto real-world scenes. AR has become increasingly popular in a wide range of applications and is now supported by most smartphones and tablets; iOS and Android, two major mobile operating systems, now support AR natively. ${ }^{5-6}$ Chemistry can also largely benefit by the highly realistic and immersive nature of this tool to visualize 3D molecular structures in the real world. Recently, a number of applications have been developed for viewing molecules in AR, primarily 
for chemistry education. ${ }^{7}$ Currently, these applications require the use of markers, such as QR code or specific patterns printed on a card, in order to place the 3D model and track its location ${ }^{8-}$ ${ }^{17}$, or the use of specialized hardware, such as AR glasses or head-mounted displays. ${ }^{18-21}$

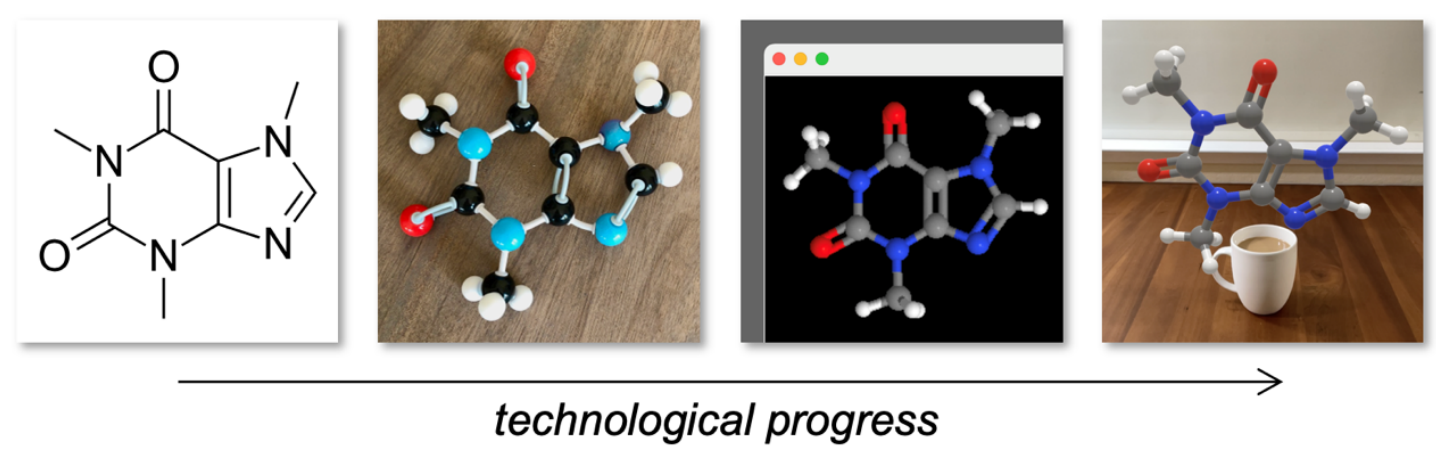

Figure 1. Visualization of caffeine in different mediums. Left to right: skeletal formula, molecular model kit, molecular modeling software, and augmented reality.

Although molecules are dynamic, three-dimensional objects, static 2D skeletal chemical structures continue to be the primary language of chemists for communicating chemical information due to their remarkably simple and intuitive nature. Visualizing 3D representation in AR directly from hand-drawn skeletal structures would thus represent a technological milestone in molecular visualization; this would provide a highly accessible way of visualizing molecules in $3 \mathrm{D}$ for researchers and students. Rather than static 2D structures on a page, the molecule would appear above the page in $3 \mathrm{D}$, providing a far richer experience for chemists.

This piece of technology relies not only on AR molecular visualization, but also demands a tool able to convert an image of a 2D chemical structure into a machine-readable format, a task known as optical chemical structure recognition. Most traditional structure recognition tools use a rule-based approach ${ }^{22-38}$ with complicated workflows, focusing only on computer-generated structures. Deep learning (DL), a new class of machine learning techniques that uses a datadriven approach, has resulted in ground-breaking performance in a huge number of tasks, from image recognition to protein folding prediction. ${ }^{39-40}$ Researchers have recently begun applying DL techniques to optical chemical structure recognition. ${ }^{41-44}$ We recently developed ChemPix, a deep learning-based algorithm that recognizes and parses photographed hydrocarbon skeletal structures. ${ }^{45}$ The algorithm can handle not only computer-generated structures, but also handdrawn structures with wobbly lines, gaps, uneven bond angles, background noise, and shadows. 
This opens up possibilities for images of hand-drawn hydrocarbon structures to be used as input for a whole host of technologies, including AR visualization.

Herein, we combine the two cutting-edge technologies of machine learning and augmented reality to develop MolAR, a mobile application which allows molecules to come to life starting from its skeletal structure. The hand-drawn hydrocarbon structure recognition feature provides a natural and accessible way of inputting molecular structures, and AR provides quick visualization of the $3 \mathrm{D}$ structures, making it effortless for users to understand how molecules of interest reside in space. Furthermore, employing an object recognition commercial package, the app also has the functionality to recognize the chemicals in objects such as food and drink, providing users the opportunity to "hunt" for molecules in their real-life environment.

\section{Results and Discussion}

MolAR is an iOS application that can be downloaded for free from Apple's App Store. ${ }^{1}$ It recognizes chemical structures directly from real-world images and dynamically presents them as 3D AR molecules in real time. The app does not require the use of markers, printouts, or specialized hardware, and there is no need to prepare 3D model files prior to use or pre-register molecules with the application. This makes MolAR an accessible visualization tool for use in chemistry laboratories and classrooms.

Figure 2 highlights one of MolAR's main functionalities. Users can photograph a hydrocarbon structure on a piece of paper or a whiteboard and visualize it in AR. The 3D molecule appears above the structure, and users can perform the pinch gesture on the phone to scale it up or down or use touch and drag to rotate and translate the model. This allows users, for example, to take a picture of a $2 \mathrm{D}$ chemical structure in the textbook they are reading and visualize it as a 3D model in a matter of seconds. Hydrocarbon structures can also be drawn directly in the app for instances when pen and paper are not handy (Figure 4a).

${ }^{1}$ https://apps.apple.com/us/app/molar/id1559504847 


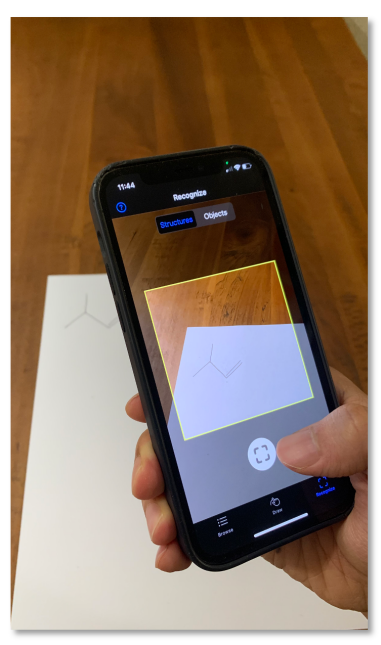

(a)

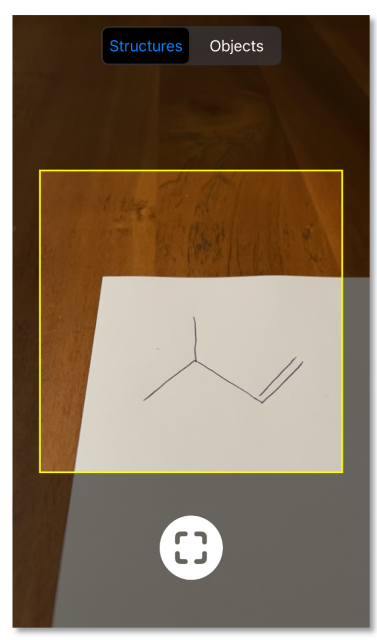

(b)

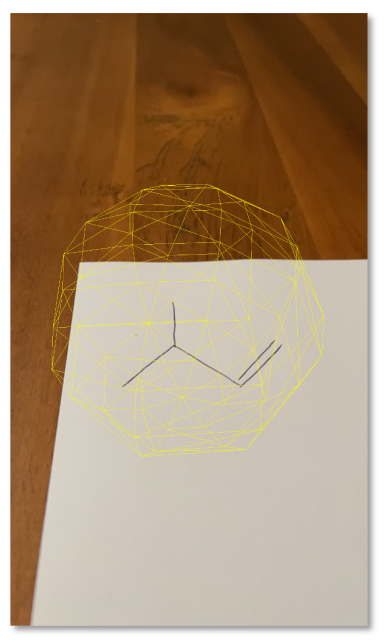

(c)

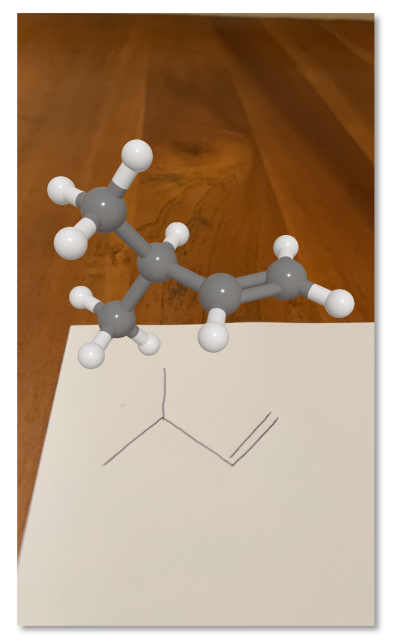

(d)

Figure 2. The user interface for chemical structure recognition. (a-b) The user takes a picture of a hydrocarbon structure, which can be hand-drawn or computer-generated. (c) The molecule is then recognized using ChemPix ${ }^{45}$, and $(\mathrm{d})$ the $3 \mathrm{D}$ molecule is shown in AR floating out of the 2D structure. See this in action at https://youtu.be/bLqkzz1vZL4.

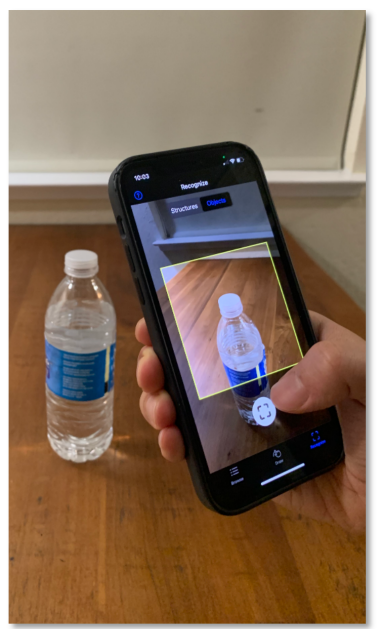

(a)

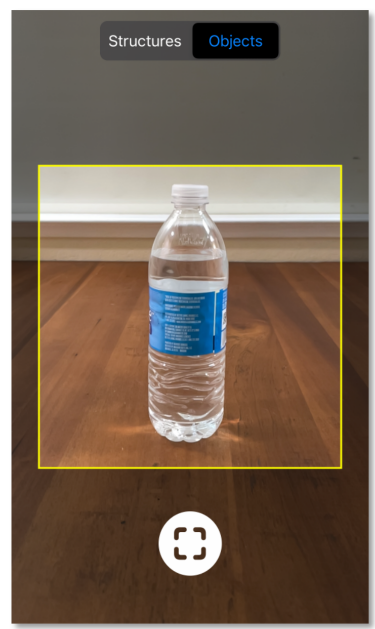

(b)

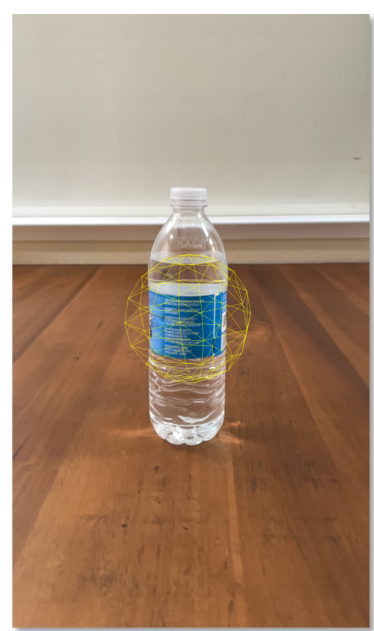

(c)

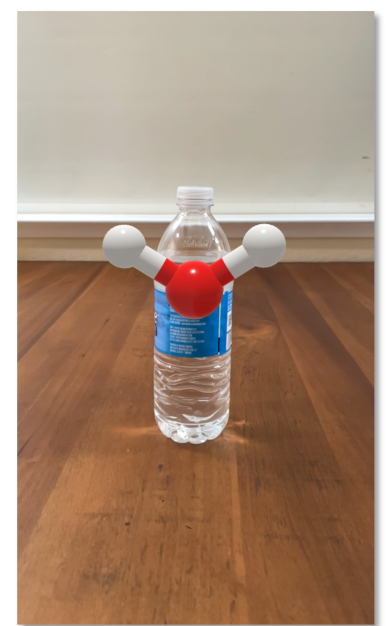

(d)

Figure 3. The user interface for object recognition. (a-b) The user takes a picture of an object. (c) The app processes the picture and then (d) shows a representative molecule of the object in AR. See this in action at https://youtu.be/bLqkzzlvZL4.

Users also have the option to "hunt" for molecules in common household objects such as food and drink and uncover representative molecules inside them (Figure 3). Objects are mapped to a characteristic molecule responsible for its flavor, color, or smell. For example, when users take a picture of coffee, the 3D model of caffeine appears above it. This can serve to gamify the app, allowing users to hunt for and learn about molecules in everyday life - in the spirit of 
"Pokémon Go" where users collect virtual creatures in real-world scenes. ${ }^{46}$ The idea is to provide the opportunity for interested children (and adults) to grasp that objects in the real world are comprised of molecules which determine their properties. The feature is designed to develop their scientific curiosity by actively learning about chemicals in a fun and engaging way.

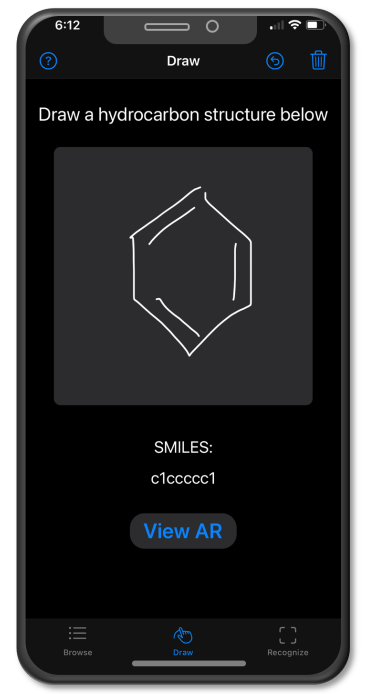

(a) Canvas for chemical structure recognition

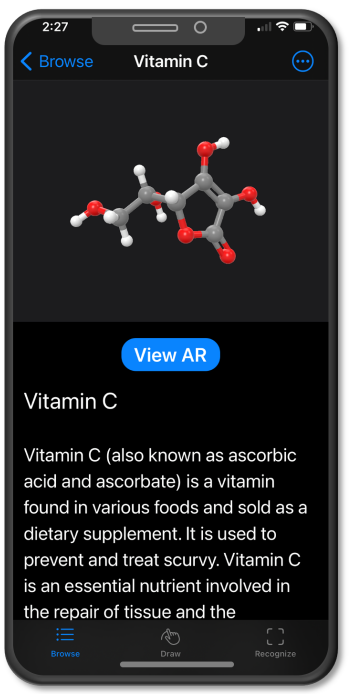

(c) Molecule description

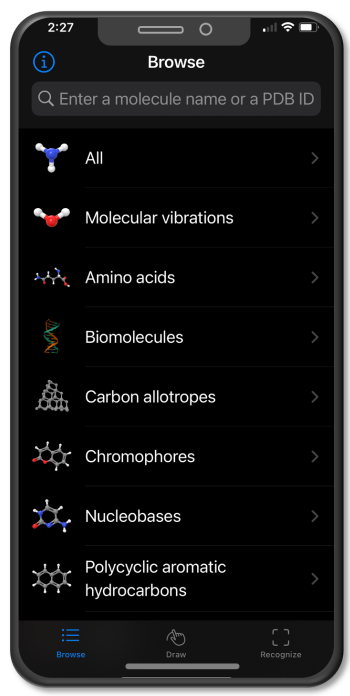

(b) Molecule gallery

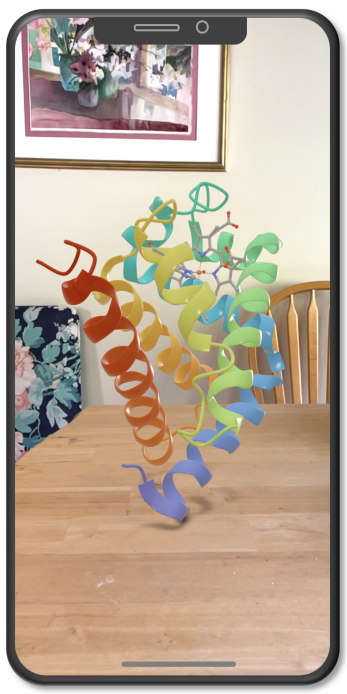

(d) Protein and macromolecule visualization

Figure 4. Other ways to input molecules. (a) Users can directly draw a hydrocarbon structure inside the app and visualize it in AR. (b) The molecule gallery lets users browse molecules and search for them by name. (c) Users can read the description of each molecule and visualize it in AR. (d) Users can visualize molecules on PubChem by entering their name or SMILES string and proteins in the Protein Data Bank by entering their PDB ID. Shown here is myoglobin (PDB ID: $1 \mathrm{MBN})$. 
To help users who are unfamiliar with chemical structures learn about molecules, the app has a gallery section where users can browse molecules and proteins of interest, read their descriptions, and visualize them in AR (Figures $4 \mathrm{~b}$ and $4 \mathrm{c}$ ). For example, users can view allotropes of carbon such as diamond, graphite, and nanotubes, and learn how different arrangements of carbon atoms in 3D space affect their properties (Figure S1). The app also allows users to visualize vibrational normal modes for a selection of molecules of educational interest such as water, ammonia, and methane. Through this feature, users can learn about vibrational motion by watching atoms animating in a real-world scene (Figure 5). Students can become familiar with the symmetry features of the vibrational modes, for example, distinguishing between symmetric and asymmetric stretching of the water molecule. A strong understanding of these normal vibrational modes is imperative in understanding spectroscopy and lends itself to class exercises such as identifying vibrations that induce a change in dipole moment.

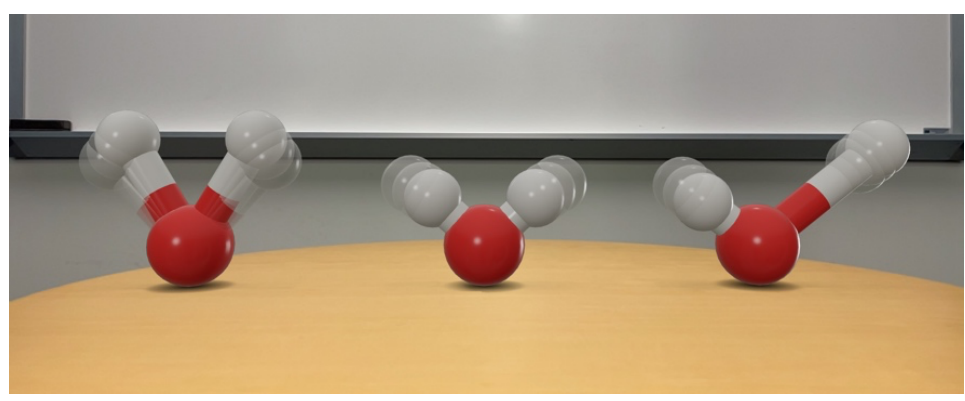

Figure 5. Molecular vibrations in AR. The app lets users visualize vibrational normal modes for a selection of molecules and watch atoms animating in a real-world scene.

Finally, users can view in AR any molecule on PubChem by typing its name or SMILES and any of the $180,000+$ proteins and biomolecules in the Protein Data Bank by entering its PDB ID (Figure $4 \mathrm{~d}$ ). The immersive interaction with a $3 \mathrm{D}$ life-size protein offers a unique way to inspect the active site, the solvent accessible channels, and the interaction among residues, helping to unveil the complex structure-function relationships occurring in large biomolecules. In addition to research applications, making visualization of protein structures accessible to the general public could aid in effectively communicating scientific viewpoints. For example, users could visualize the SARS-CoV-2 spike protein floating in their living rooms on their phone screens, which may encourage non-chemists to engage more in science and hence be more open to scientific viewpoints. This has the potential to facilitate science-driven policy. 
The MolAR application provides a new visualization tool for use in chemistry laboratories and classrooms. In education, we aim to help students become familiar with the threedimensional spatial arrangement of atoms in molecules. A recent study by Rahmawati et al. found that the use of 3D virtual representations in classrooms can develop students' spatial abilities, resulting in a better understanding of chemical concepts. ${ }^{47}$ With this in mind, MolAR serves as an immersive training application to help students master mental visualization of the 3D structures of molecules. Since the app does not require specialized hardware, it is easily accessible to potential users. Moreover, the software does not need a desktop computer or laptop, adding a high level of portability and convenience to the tool. In research, MolAR provides barrierless visualization of protein structures and analysis of molecular arrangements to aid in the understanding of chemical behavior.

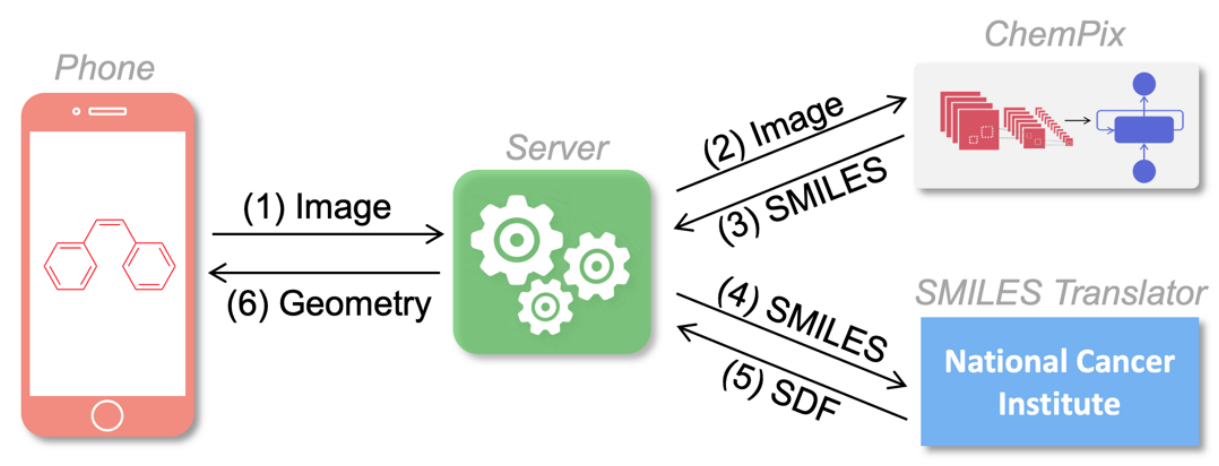

Figure 6. The workflow for transforming a hand-drawn chemical structure into a 3D AR model. (1) The app sends the image of the structure to the server, which then (2-3) feeds the image to ChemPix ${ }^{45}$ to predict the SMILES representation. (4-5) The server then sends the SMILES to the Online SMILES Translator by the National Cancer Institute to obtain the SDF data. (6) The server converts the SDF to a list of geometric primitives and returns it to the app.

The MolAR workflow for transforming a hand-drawn hydrocarbon structure into a 3D AR model is summarized in Figure 6. When the user takes a picture of a chemical structure, the app starts tracking the position of the structure so that it can place the model there later. The picture is sent to a server, which feeds the image to ChemPix ${ }^{45}$ to predict the SMILES representation of the structure. The server then sends the SMILES to the Online SMILES Translator by the National Cancer Institute to obtain an SDF file with the molecular structure. ${ }^{48}$ The server converts the SDF to a list of geometric primitives and returns it to the app. Finally, the app converts this list to a USDZ file, a format for AR models on iOS devices, and places the 
molecular structure above the hand-drawn image. Further technical details are provided in the Methods section and Supplementary Information.

The workflow for object recognition is similar. When the user takes a picture of an object, the app also sends the picture to the server. The server uses Google Cloud Vision API and Amazon Rekognition for object detection. ${ }^{49-50}$ We have developed a database that maps common objects to molecules; for example, coffee is mapped to caffeine, and carrots are mapped to carotene (Table S1). The server then sends the USDZ file for the molecular structure corresponding to the photographed object to the app. As in hand-drawn hydrocarbon workflow, the app tracks the object during the computation and presents the $3 \mathrm{D}$ chemical structure above it.

\section{Conclusions and outlook}

In conclusion, we developed MolAR, a mobile application applying state-of-the-art techniques in machine learning and augmented reality. While the advent of computer graphics led chemists to interact with molecules in a simplified cartoon representation consisting of spheres (atoms) and cylinders (bonds), the advancement of AR technology allows chemists to visualize and interact with life-size molecules as if they existed in the real world. The combination of machine learning with AR visualization pushes the limit further still, allowing the conversion of a hand-drawn hydrocarbon to a 3D AR representation.

The app serves as a building block which can be directly connected to a whole host of additional tools. In the future, we plan to incorporate dynamic motion, chemical reactions, and computation of quantum mechanical properties. This will allow the visualization of orbitals and calculation of spectra. We also plan to link MolAR to our recent work of ChemVox that performs voice-activated quantum chemistry. ${ }^{51}$ The combination of chemical structure and speech input offers a powerful combination; one could imagine drawing a structure of a molecule and asking for the app to "compute the dipole moment of this molecule in ethanol" for example. We are in the process of extending the ChemPix hydrocarbon recognition framework to also recognize molecules with heteroatoms.

Overall, MolAR is the latest example of how cutting-edge technologies can enhance scientific research and education, offering another valuable tool in the scientist's current arsenal. 


\section{Methods}

The MolAR app is written using the Swift programming language ${ }^{52}$ and Apple's iOS SDK ${ }^{53}$, a software development kit for iPhone and iPad. It uses ARKit ${ }^{54}$, part of the iOS SDK, to implement augmented reality. ARKit has features for AR such as device motion tracking and scene processing. The app communicates with a web server whose main functionality is to recognize objects or chemical structures in an image. The server is written in Node.js. ${ }^{55} \mathrm{We}$ describe the method in detail below.

$A R$ tracking. When the user takes a picture of an object or a chemical structure, the app places a virtual anchor at the target. The virtual anchor allows the app to track the location of the target as the user moves while the app is processing the picture. Once the app receives the 3D structure from the server, the app places it at the anchor.

Image to SMILES. The server feeds the image it receives to the image-to-SMILES neural network described by Weir et al. ${ }^{45}$ The image of the chemical structure is encoded with a convolutional neural network $(\mathrm{CNN})$ which is then decoded using a long short-term memory (LSTM) network to a SMILES representation. An ensemble model is applied, comprised of five trained neural networks that each cast one vote for the predicted SMILES string. The highest voted SMILES is output by the model.

SMILES to SDF. We use the Online SMILES Translator by the National Cancer Institute to convert SMILES to SDF. The SDF data of a molecule contains the 3D coordinates of each atom in the molecule and the type of the bond between pairs of atoms.

SDF to geometric primitives. In this step, atoms and bonds in the SDF data are converted to spheres and cylinders. Atoms are represented by spheres with the color according to the CPK coloring and the radius according to the van der Waals radius scaled down by a constant. Bonds are represented by cylinders, each with the color of the sphere it connects.

Geometric primitives to USDZ. USDZ is a file format created by Pixar for interchange of 3D models. ${ }^{56}$ iOS has a built-in viewer that can show USDZ files in AR. The app generates USDZ files from geometric primitives according to the file format specification. ${ }^{57}$ We choose to generate USDZ files on device rather than on the server because the USDZ file size is much larger than that of the geometric primitives.

Visualizing molecular vibrations. The USDZ file format supports keyframe animations. ${ }^{58}$ Each geometric object can be translated, rotated, or scaled by specifying the transformation 
matrices at different points in time. To visualize molecular vibrations, we animate atoms and bonds separately. The sphere for each atom is translated according to its trajectory. The cylinder for each bond is translated, rotated, and scaled to maintain the connection between the atoms.

Visualizing Proteins. When visualizing a protein, the app fetches the CIF data from the Protein Data Bank and then uses Mol*59, an open-source macromolecular toolkit, to generate 3D coordinates of the protein. We use the cartoon representation for small or medium proteins and the Gaussian surface representation for large proteins to improve the display performance.

\section{Data availability}

The source code of MolAR can be found at https://github.com/mtzgroup/molar.

\section{Acknowledgements}

This work was supported by the Office of Naval Research (N00014-18-1-2659 and N00014-18$1-2624)$. 


\section{References}

1. Pray, L., Discovery of DNA structure and function: Watson and Crick. Nature Education 2008, 1 (1).

2. Watson, J., The Double Helix: A Personal Account of the Discovery of the Structure of $D N A$. Atheneum: New York, 1968.

3. Francoeur, E., The Forgotten Tool: The Design and Use of Molecular Models. Social Studies of Science 1997, 27 (1), 7-40.

4. Olson, A. J., Perspectives on Structural Molecular Biology Visualization: From Past to Present. J Mol Biol 2018, 430 (21), 3997-4012.

5. Apple. Augmented Reality. https://developer.apple.com/augmented-reality/ (accessed August 16, 2021).

6. Google. Build new augmented reality experiences that seamlessly blend the digital and physical worlds. (accessed August 16, 2021).

7. Jiménez, Z. A., Teaching and Learning Chemistry via Augmented and Immersive Virtual Reality. In Technology Integration in Chemistry Education and Research (TICER), American Chemical Society: 2019; Vol. 1318, pp 31-52.

8. Cai, S.; Wang, X.; Chiang, F.-K., A case study of Augmented Reality simulation system application in a chemistry course. Computers in Human Behavior 2014, 37, 31-40.

9. Chen, M.; Liao, B. In Augmented Reality Laboratory for High School Electrochemistry Course, 2015 IEEE 15th International Conference on Advanced Learning Technologies, 6-9 July 2015; 2015; pp 132-136.

10. Hou, H.; Lin, Y. In The Development and Evaluation of an Educational Game Integrated with Augmented Reality and Virtual Laboratory for Chemistry Experiment Learning, 2017 6th IIAI International Congress on Advanced Applied Informatics (IIAI-AAI), 9-13 July 2017; 2017; pp 1005-1006.

11. Iordache, D. D.; Pribeanu, C.; Balog, A., Influence of specific AR capabilities on the learning effectiveness and efficiency. Studies in Informatics and Control 2012, 21 (3), 233-240.

12. L, N. S. K.; Chavan, P. S.; Murthy, S. In StereoChem: Augmented Reality 3D Molecular Model Visualization App for Teaching and Learning Stereochemistry, 2018 IEEE 18th International Conference on Advanced Learning Technologies (ICALT), 9-13 July 2018; 2018; pp 252-256.

13. Maier, P.; Klinker, G. In Augmented chemical reactions: An augmented reality tool to support chemistry teaching, 2013 2nd Experiment@ International Conference (exp.at'13), 18-20 Sept. 2013; 2013; pp 164-165.

14. Nachairit, A.; Srisawasdi, N. In Using mobile augmented reality for chemistry learning of acid-base titration: correlation between motivation and perception, Proceedings of the 23rd International Conference on Computers in Education, Asia-Pacific Society for Computers in Education Hangzhou: 2015; pp 519-528.

15. Plunkett, K. N., A Simple and Practical Method for Incorporating Augmented Reality into the Classroom and Laboratory. Journal of Chemical Education 2019, 96 (11), 2628-2631.

16. Qassem, L. M. M. S. A.; Hawai, H. A.; AlShehhi, S.; Zemerly, M. J.; Ng, J. W. P. In AIREDUTECH: Augmented immersive reality (AIR) technology for high school Chemistry education, 2016 IEEE Global Engineering Education Conference (EDUCON), 10-13 April 2016; 2016; pp 842-847.

17. Singhal, S.; Bagga, S.; Goyal, P.; Saxena, V., Augmented chemistry: Interactive education system. International Journal of Computer Applications 2012, 49 (15). 
18. Peterson, C. N.; Tavana, S. Z.; Akinleye, O. P.; Johnson, W. H.; Berkmen, M. B., An idea to explore: Use of augmented reality for teaching three-dimensional biomolecular structures. Biochemistry and Molecular Biology Education 2020, 48 (3), 276-282.

19. Uselton, M. MoleculAR Geometry: Chemical Visualizations in Augmented Reality. Honors College of Middle Tennessee State University, 2020.

20. Zheng, M.; Waller, M. P., ChemPreview: an augmented reality-based molecular interface. Journal of Molecular Graphics and Modelling 2017, 73, 18-23.

21. Zhu, B.; Feng, M.; Lowe, H.; Kesselman, J.; Harrison, L.; Dempski, R. E., Increasing Enthusiasm and Enhancing Learning for Biochemistry-Laboratory Safety with an AugmentedReality Program. Journal of Chemical Education 2018, 95 (10), 1747-1754.

22. Algorri, M.-E.; Zimmermann, M.; Friedrich, C. M.; Akle, S.; Hofmann-Apitius, M. In Reconstruction of chemical molecules from images, 2007 29th Annual International Conference of the IEEE Engineering in Medicine and Biology Society, IEEE: 2007; pp 4609-4612.

23. Beard, E. J.; Cole, J. M., ChemSchematicResolver: A toolkit to decode 2D chemical diagrams with labels and R-groups into annotated chemical named entities. Journal of Chemical Information and Modeling 2020, 60 (4), 2059-2072.

24. Casey, R.; Boyer, S.; Healey, P.; Miller, A.; Oudot, B.; Zilles, K. In Optical recognition of chemical graphics, Proceedings of 2nd International Conference on Document Analysis and Recognition (ICDAR'93), IEEE: 1993; pp 627-631.

25. Contreras, M. L.; Allendes, C.; Alvarez, L. T.; Rozas, R., Computational perception and recognition of digitized molecular structures. Journal of chemical information and computer sciences 1990, 30 (3), 302-307.

26. Filippov, I. V.; Nicklaus, M. C., Optical structure recognition software to recover chemical information: OSRA, an open source solution. ACS Publications: 2009.

27. Frasconi, P.; Gabbrielli, F.; Lippi, M.; Marinai, S., Markov logic networks for optical chemical structure recognition. Journal of Chemical Information and Modeling 2014, 54 (8), 2380-2390.

28. Gkoutos, G. V.; Rzepa, H.; Clark, R. M.; Adjei, O.; Johal, H., Chemical machine vision: automated extraction of chemical metadata from raster images. Journal of chemical information and computer sciences 2003, 43 (5), 1342-1355.

29. Ibison, P.; Jacquot, M.; Kam, F.; Neville, A.; Simpson, R. W.; Tonnelier, C.; Venczel, T.; Johnson, A. P., Chemical literature data extraction: the CLiDE Project. Journal of Chemical Information and Computer Sciences 1993, 33 (3), 338-344.

30. McDaniel, J. R.; Balmuth, J. R., Kekule: OCR-optical chemical (structure) recognition. Journal of chemical information and computer sciences 1992, 32 (4), 373-378.

31. Ouyang, T. Y.; Davis, R. In Recognition of hand drawn chemical diagrams, AAAI, 2007; pp 846-851.

32. Park, J.; Rosania, G. R.; Shedden, K. A.; Nguyen, M.; Lyu, N.; Saitou, K., Automated extraction of chemical structure information from digital raster images. Chemistry Central Journal 2009, 3 (1), 1-16.

33. Park, J.; Saitou, K.; Rosania, G. In Image-based automated chemical database annotation with ensemble of machine-vision classifiers, 2010 IEEE International Conference on Automation Science and Engineering, IEEE: 2010; pp 168-173.

34. Rajan, K.; Brinkhaus, H. O.; Zielesny, A.; Steinbeck, C., A review of optical chemical structure recognition tools. Journal of Cheminformatics 2020, 12 (1), 1-13. 
35. Rosania, G. R.; Crippen, G.; Woolf, P.; Shedden, K., A cheminformatic toolkit for mining biomedical knowledge. Pharmaceutical Research 2007, 24 (10), 1791-1802.

36. Sadawi, N. M.; Sexton, A. P.; Sorge, V. In Chemical structure recognition: a rule-based approach, Document Recognition and Retrieval XIX, International Society for Optics and Photonics: 2012; p 82970E.

37. Tharatipyakul, A.; Numnark, S.; Wichadakul, D.; Ingsriswang, S. In ChemEx: information extraction system for chemical data curation, BMC bioinformatics, Springer: 2012; pp 1-11.

38. Valko, A. T.; Johnson, A. P., CLiDE Pro: the latest generation of CLiDE, a tool for optical chemical structure recognition. Journal of Chemical Information and Modeling 2009, 49 (4), 780-787.

39. Jumper, J.; Evans, R.; Pritzel, A.; Green, T.; Figurnov, M.; Ronneberger, O.; Tunyasuvunakool, K.; Bates, R.; Žídek, A.; Potapenko, A.; Bridgland, A.; Meyer, C.; Kohl, S. A. A.; Ballard, A. J.; Cowie, A.; Romera-Paredes, B.; Nikolov, S.; Jain, R.; Adler, J.; Back, T.; Petersen, S.; Reiman, D.; Clancy, E.; Zielinski, M.; Steinegger, M.; Pacholska, M.; Berghammer, T.; Bodenstein, S.; Silver, D.; Vinyals, O.; Senior, A. W.; Kavukcuoglu, K.; Kohli, P.; Hassabis, D., Highly accurate protein structure prediction with AlphaFold. Nature 2021.

40. Krizhevsky, A.; Sutskever, I.; Hinton, G. E., ImageNet classification with deep convolutional neural networks. Commun. ACM 2017, 60 (6), 84-90.

41. Oldenhof, M.; Arany, A.; Moreau, Y.; Simm, J., ChemGrapher: Optical Graph Recognition of Chemical Compounds by Deep Learning. Journal of Chemical Information and Modeling 2020, 60 (10), 4506-4517.

42. Rajan, K.; Zielesny, A.; Steinbeck, C., DECIMER: towards deep learning for chemical image recognition. Journal of Cheminformatics 2020, 12 (1), 65.

43. Staker, J.; Marshall, K.; Abel, R.; McQuaw, C. M., Molecular Structure Extraction from Documents Using Deep Learning. Journal of Chemical Information and Modeling 2019, 59 (3), 1017-1029.

44. Vision Arcanum. InkToMolecule online. https://visionarcanum.com/ink2mol/ (accessed August 16, 2021).

45. Weir, H.; Thompson, K.; Woodward, A.; Choi, B.; Braun, A.; Martínez, T. J., ChemPix: automated recognition of hand-drawn hydrocarbon structures using deep learning. Chemical Science 2021, 12 (31), 10622-10633.

46. Plagge, K. Pokemon Go Review. https://www.ign.com/articles/2016/07/13/pokemon-goreview (accessed August 16, 2021).

47. Rahmawati, Y.; Dianhar, H.; Arifin, F., Analysing Students' Spatial Abilities in Chemistry Learning Using 3D Virtual Representation. Education Sciences 2021, 11 (4), 185.

48. National Cancer Institute. Online SMILES Translator. https:/cactus.nci.nih.gov/translate/index.html (accessed August 16, 2021).

49. Amazon Web Services. Detecting labels. https://docs.aws.amazon.com/rekognition/latest/dg/labels.html (accessed August 16, 2021).

50. Google. Detect Labels. https://cloud.google.com/vision/docs/labels (accessed August 16, 2021).

51. Raucci, U.; Valentini, A.; Pieri, E.; Weir, H.; Seritan, S.; Martínez, T. J., Voice-controlled quantum chemistry. Nature Computational Science 2021, 1 (1), 42-45.

52. Apple. Swift. https://developer.apple.com/swift/ (accessed August 16, 2021).

53. Apple. iOS. https://developer.apple.com/ios/ (accessed August 16, 2021).

54. Apple. ARKit. https://developer.apple.com/documentation/arkit (accessed August 16, 2021). 
55. OpenJS Foundation. Node.js. https://nodejs.org/ (accessed August 16, 2021).

56. Pixar Animation Studios. Introduction to USD. https://graphics.pixar.com/usd/docs/Introduction-to-USD.html (accessed August 16, 2021).

57. Pixar Animation Studios. Usdz File Format Specification. https://graphics.pixar.com/usd/docs/Usdz-File-Format-Specification.html (accessed August 16, 2021).

58. Pixar Animation Studios. Transformations, Time-sampled Animation, and Layer Offsets. https://graphics.pixar.com/usd/docs/567231471.html (accessed August 16, 2021).

59. Sehnal, D.; Bittrich, S.; Deshpande, M.; Svobodová, R.; Berka, K.; Bazgier, V.; Velankar, S.; Burley, S. K.; Koča, J.; Rose, A. S., Mol* Viewer: modern web app for 3D visualization and analysis of large biomolecular structures. Nucleic Acids Research 2021, 49 (W1), W431-W437. 